\title{
Excessively tilted fiber grating sensors
}

\author{
Sun Yuezhen, Yan Zhijun, Zhou Kaiming, Luo Binbin, Jiang Biqiang, Mou Chengbo, Sun Qizhen and \\ Zhang Lin. \\ Invited Paper
}

\begin{abstract}
The development of excessively tilted fiber gratings (Ex-TFGs) provides a new type of sensing device with high refractive index (RI) sensitivity, low thermal crosstalk and vector sensing property. Due to the asymmetric grating structure of ExTFG, the light in the core is coupled into high order forwardpropagating cladding modes and split into two orthogonal polarization states, resulting in dual-peak resonances in the transmission spectrum. The Ex-TFG also exhibits a non-circularly symmetrical near field distribution, which endows an exceptional orientation sensing capability. Benefitting from the unique mode coupling behavior, Ex-TFGs have been studied and developed for many different sensing applications, such as polarization dependent torsion and loading sensors, vector accelerometer and magnetometer, and a variety of low thermal crosstalk bio/chemical sensors.
\end{abstract}

This paper will review the recent study and development of ExTFGs in terms of mode coupling mechanism, fabrication method, transmission and sensor characteristics and the novel applications in sensing areas.

Index Terms - Optical fiber grating, mode coupling in excessively asymmetric structure, refractive index sensing, orientation sensing, biosensing.

\section{INTRODUCTION}

The transverse holographic method [1] developed in 1989 opened a new door for the development of optical fiber grating (OFG) technology. Since then, researchers have proposed a range of novel OFGs. Different structures of OFGs manipulate the mode coupling behavior of light transmitted in the fiber through periodic core refractive index modulation. Generally speaking, the mode coupling in an OFG may include core, cladding and radiation modes. Different OFG structures possess different mode coupling mechanisms, which depend on the period and tilt angle of the grating [2-4]. By designing the period and tilt angle, OFGs can exhibit different mode power coupling and transmission property, which can generate a variety of light manipulation functions and be utilized in applications in fiber communication systems [5], fiber lasers [6] and fiber sensing fields [7].

Fiber Bragg gratings (FBGs) with a typical period of few hundreds of nanometers [8] only allow coupling the light from the forward-propagating core mode to the backwardpropagating one, resulting in reflection type of light filters [910]. Long period gratings (LPGs) with hundreds of micrometers period are capable of coupling light from forward-propagating core mode to the forward-propagating cladding modes, generating loss bands in transmission [11]. Attributing to the cladding mode coupling, LPGs are intrinsically sensitive to the surrounding-medium refractive index (SRI) change, and more applied in bio/chemical sensing applications [12]. In comparison with FBGs and LPGs of normal structure, tilted fiber gratings (TFGs) possess unique polarization characteristics due to the modulated refractive index tilted with respect to the fiber axis. According to the mode coupling behavior, TFGs are classified as small angle tilted fiber Bragg grating (TFBG), radiation tilted fiber gratings (RTFG) and excessively tilted fiber grating (Ex-TFG). The transmission light is coupled from the forward-propagating core mode to the backward-propagating cladding modes in a TFBG, to the radiation modes in an RTFG and to the forward-propagating cladding modes in an Ex-TFG, respectively. Considerable research work on TFGs has been reported previously [13-14]. The most representative TFBGs work is the TFBGs based surface plasmon resonance (SPR) excitation for bio/chemical sensing, which is proposed and developed by J. Albert and T. Guo et al. [15-18]. For RTFGs, Westbrook et al. were the first to demonstrate a $45^{\circ}$-TFG based in-fiber polarimeter in 2000 [19], and since then, $45^{\circ}$-TFGs have been developed as high polarization extinction ratio in-fiber polarizer $[20,21]$ and diffractive grating [22], of which the unique single polarization coupling property has promoted the $45^{\circ}$-TFGs for applications in mode-locking fiber lasers, optical fiber coherent tomography, optical wireless communication and spectral code imaging system [23-25].

In 2006, the research group at Aston University proposed a fiber grating structure with $>80^{\circ}$ tilt angle with respect to the normal fiber axis and relatively short period (tens micrometer), named Ex-TFG. Such Ex-TFG shows a series of orthogonal polarization dual-peaks in the transmission spectrum and exhibits high SRI sensitivity with low temperature crosstalk [26]. In 2014, Mou et al. [27] have published a book chapter about sensing properties of Ex-TFGs, which only includes some basic physical applications. In this paper, we will present a comprehensive review on the more recent study and development of theory, fabrication, characteristics and applications of Ex-TFGs, which would provide more insights to the Ex-TFGs and promote a more wide range of applications in utilizing such unique optical fiber grating devices.

\section{THEORY AND FABRICATION OF EX-TFG}

An Ex-TFG has an equivalent pile of periodically slanted plates that are inclined more than 66.9 degrees with respect to the normal optical fiber axis [28], as schematically shown in Fig.1.Such large angle titled structure of Ex-TFG favors the forward propagating core mode coupling into the forward propagating cladding modes, and its period, typically around tens of microns, supports excitation of higher order cladding modes. Due to its slanted structure, an Ex-TFG is intrinsically birefringent with assigned fast and slow axis as depicted in Fig. 
1.

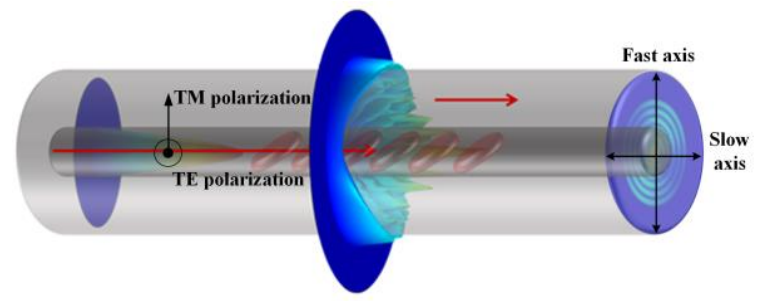

Fig. 1. The schematic diagram of an Ex-TFG [29].

\section{A. Mode coupling theory of Ex-TFG}

Usually, the mode coupling strength between core and cladding modes under weak waveguide approximation is determined by the coupling coefficient, which can be expressed as [4]:

$$
\begin{array}{rl}
g_{l m-01}^{c l}=A_{g}^{c l} \int_{0}^{a} & r d r J_{l-1}\left(2 r K_{g} \sin \theta\right) \\
& \times J_{l-1}\left(u_{l m} r\right) J_{0}\left(u_{0} r\right)
\end{array}
$$

where $A_{g}^{c l}$ is the amplitude of the cladding mode, $a$ is the core radius, $J$ is the Bessel function of the first kind, $K_{g}$ is the wave number of TFG, $l m$ represents the hybrid mode, $u_{l m}{ }^{2}=$ $(2 \pi / \lambda)^{2}\left(n_{1}{ }^{2}-n_{\text {efflm }}{ }^{2}\right)\left(n_{1}\right.$ and $n_{\text {efflm }}$ are the refractive index of core material and effective index of cladding mode, respectively), and $\theta$ is the tilt angle of the grating.

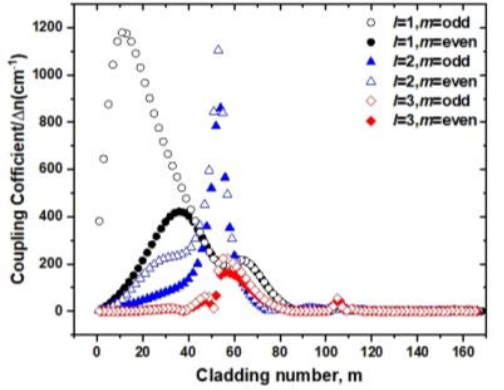

Fig. 2. Coupling coefficient of different cladding modes with $l=1,2,3$ in an Ex-TFG with $80^{\circ}$ tilt angle [29].

The calculated coupling coefficients of different cladding modes of an Ex-TFG with $80^{\circ}$ tilt angle are plotted in Fig. 2, which show that the mode coupling of Ex-TFG only occurs as azimuthal mode number $l>0$ [4, 30-32]. The largest coupling coefficient of $\mathrm{LP}_{1 \mathrm{~m}}$ is around $10^{\text {th }}$ cladding mode, and the largest for $\mathrm{LP}_{2 \mathrm{~m}}$ is at the order of $52^{\text {nd }}$, and as increasing of mode order $\left(>80^{\text {th }}\right)$, the coupling coefficient value reduces almost to 0 . It is also clear from the figure, the coupling coefficient of $\mathrm{LP}_{2 \mathrm{~m}}$ cladding mode is always smaller than that of $\mathrm{LP}_{1 \mathrm{~m}}$, until the mode order is beyond $48^{\text {th }}$. Generally, Ex-TFGs have relatively smaller period when compared to a standard LPG, around tens micrometers, and in most applications, only the first 40 orders cladding modes are used, which are mainly the $\mathrm{LP}_{l m}$ modes, according to the theoretical and simulated results in $\operatorname{Ref}[33,34]$.

\section{B. Numerical analysis}

Compared to LPG, Ex-TFG exhibits the same forwardpropagating mode coupling behavior, but the critical difference is that the tilted grating structure induces a polarizationdependent mode coupling property. So, Ex-TFG sometimes is also called tilted long period fiber grating [33]. To qualitatively simulate the coupling behavior of EX-TFG, the weak waveguide approximation was employed in the simulation. The $\mathrm{LP}_{1 \mathrm{~m}}$ modes in an Ex-TFG are the degenerated TM and TE modes, which are a pair of orthogonally linearly polarized modes (polarization direction shown in Fig.1). The eigenvalue equations for TMom and TEom are given as (2) and (3), respectively [35].

$$
\begin{gathered}
\frac{J_{1}(u)}{u J_{0}(u)}+(1-2 \Delta n) \frac{K_{1}(w)}{w K_{0}(w)}=0 \\
\frac{J_{1}(u)}{u J_{0}(u)}+\frac{K_{1}(w)}{w K_{0}(w)}=0
\end{gathered}
$$

Where $\Delta n$ is the refractive index difference between the cladding and air; $u=(2 \pi r)^{2}\left(n_{c l}{ }^{2}-n_{\text {cleff }}{ }^{2}\right)^{1 / 2} / \lambda$ and $w=(2 \pi r)^{2}\left(n_{\text {cleff }}{ }^{2}-n_{\text {air }}{ }^{2}\right)^{1 / 2} / \lambda(r$ is the radius of fiber cladding; $n_{\text {cleff }}, n_{c l}$, and $n_{\text {air }}$ are the effective index of cladding mode, the refractive index of cladding material and air, respectively.

The phase matching condition for an Ex-TFG can be expressed as (4),

$$
\lambda=\left(n_{\text {eff }}^{c o}-n_{\text {eff }}^{c l, m, T E / T M}\right) \frac{\Lambda_{G}}{\cos \theta}
$$

Where $\lambda$ is the resonance wavelength; $n_{e f f}^{c o}$ is the effective index of core mode; $n_{e f f}^{c l, m, T E / T M}$ is the effective index of $m^{\text {th }}$ cladding mode of TE or TM polarization; $\Lambda_{G}$ is the normal period of grating; $\theta$ is the tilted angle of the grating.

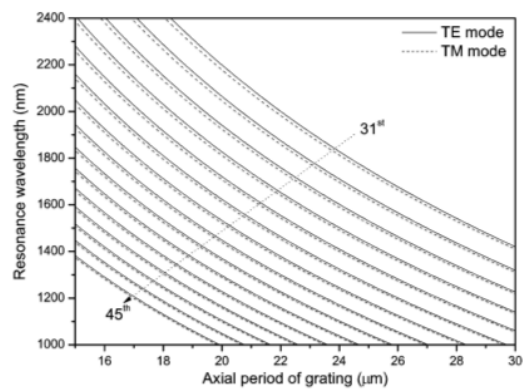

Fig. 3. Simulated resonance wavelength versus the axial period of Ex-TFG with TE (solid line) and TM (dash line) modes from $31^{\text {th }}$ to $45^{\text {th }}$ orders [35].

By using (2), (3) and (4), the resonance wavelength of ExTFG can be determined by the effective mode indices of core and cladding and the axial period of grating. By applying (4), the phase matching curve of Ex-TFG is plotted in Fig. 3, showing a series of TM and TE modes in pairs under the same cladding mode order. In the experiment, the axial period of ExTFG is generally around tens micrometer, in which the mode coupling mainly happens between the core mode and the high order cladding mode around 30th to 40th cladding mode, where the resonant wavelength of TM mode is shorter than that of TE mode.

\section{Fabrication method of Ex-TFG}

At present, the phase mask method [36-37] is the most widely used inscription technique for the fabrication of FBGs and TFBGs with sub-micrometer periods. For gratings with 
hundreds of micrometers period, such as LPGs, they are usually fabricated by the point-by-point inscription method. However, for an Ex-TFG, due to the limitation of diffraction of phase mask and tilted grating structure, the inscription methods mentioned above are not suitable.
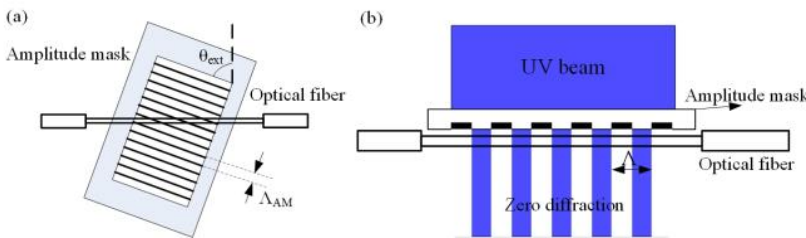

Fig. 4. Schematic of Ex-TFG inscription: (a) the front view and (b) the top view of amplitude mask with 0 order diffraction inside the fiber core [35].

Consequently, the amplitude mask method is adopted for ExTFG inscription [35]. During the inscription process, the amplitude mask is tilted at a certain angle in front of the fiber, and the grating structure is introduced by the 0 -order diffraction beam (shown in Fig. 4a and 4b). The relationship of tilt angle and period between amplitude mask and grating has been described in Ref. [35].

\section{Spectra characteristics of Ex-TFG}

Yan et al. have investigated the transmission property of ExTFG in detail [35], from which the transmission spectra of an Ex-TFG with $81^{\circ}$ tilt angle and $28 \mu \mathrm{m}$ axial period are studied and its characteristic spectrum is shown in Fig. 5a. From the figure, we clearly see that there is a series of dual-peak resonances in the range from $1300 \mathrm{~nm}$ to $1700 \mathrm{~nm}$, of which the TM peak is at shorter and TE peak is at longer wavelength side.
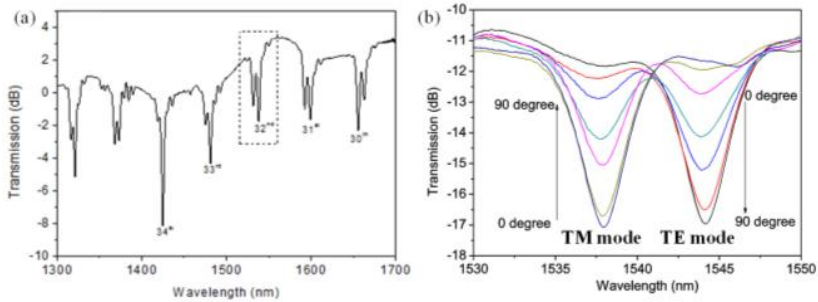

Fig. 5. The transmission spectra of an Ex-TFG with $81^{\circ}$ tilt angle: (a) a series of dual-peak resonances from 1300 to $1700 \mathrm{~nm}$ and (b) zoomed one pair of dual-peaks measured by linear polarization light with different azimuth angles with respect to the fast axis of grating [35].

Furthermore, by rotating linearly polarized light launched into the grating, the strength of dual peaks alternatively changes as seen in Fig.5b. When the polarization axis of light is parallel with the fast axis of Ex-TFG, the TM peak is at the strongest and the TE peak almost ceased, and then as rotating the polarization of incident light, the TM peak gradually reduces and TE peak arises, clearly demonstrating the polarization dependent spectral property of an Ex-TFG. According to the simulation in Ref. [35], this Ex-TFG will excite the $30^{\text {th }}-36^{\text {th }}$ cladding modes in the near infrared region, which are most suitable for communication, sensing and laser applications.

\section{SENSING CHARACTERIZATION OF EX-TFG}

Due to the high order cladding mode excitation, Ex-TFGs have shown higher RI responsivity to aqueous and lower temperature sensitivity than LPGs. The sensitivity expression of an Ex-TFG under external perturbation can be expressed as:

$$
\Delta \lambda=\gamma(\Gamma+\alpha) \lambda
$$

Where $\gamma$ is waveguide dispersion; $\Gamma$ is the environmental dependence of waveguide dispersion, such as RI and temperature (the detailed derivation process described in [38]); $\alpha$ is the material expansion coefficient.

From (4) and (5), the sensitivity of an Ex-TFG to the surrounding medium could be calculated in terms of the cladding mode order and titled angle, and the detailed discussion will be given in the following sections.

\section{A. Refractive index sensing characteristics}

Mou et al. have initially investigated the RI sensing characteristics for a typical Ex-TFG [39], showing the RI sensitivity of TM mode $(291.5 \mathrm{~nm} / \mathrm{RIU})$ is slightly higher than that $(252.5 \mathrm{~nm} / \mathrm{RIU})$ of TE mode (see in Fig. 6a), and as increasing the SRI, the resonance peaks of TM and TE cladding modes merged into one peak, as shown in Fig. $6 \mathrm{~b}$.
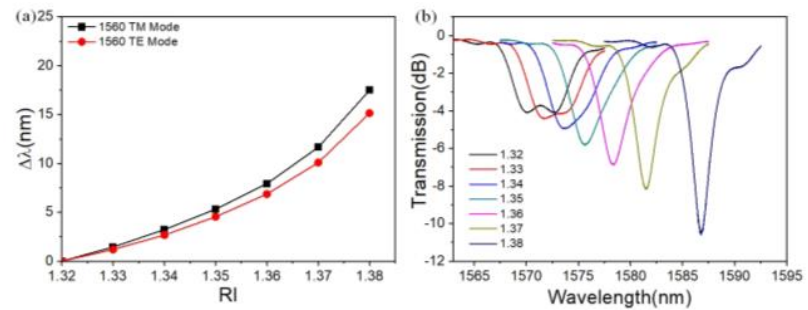

Fig. 6. (a) The transmission spectrum of Ex-TFG with dual peak under different surrounding RI; (b) wavelength shift of TM and TE mode versus different RIs and eventually merging into one peak [39].

\section{1) Mode dependent RI sensitivity}

More recently, Yan et al. have conducted a more comprehensive study and experimentally and theoretically analyzed the RI sensing characteristics of Ex-TFGs, revealing that the RI sensitivity depends on the order number of cladding mode [38]. The RI sensitivity of Ex-TFG shows a nonlinear trend, and when the SRI value approaches the effective index of cladding mode, the RI sensitivity is at the highest. They then further investigated the RI response of different order cladding modes at the resonance wavelength around $1550 \mathrm{~nm}$ by fabricating Ex-TFGs with different axial period. The results show that the lower the cladding mode order, the higher the effective mode index, but lower RI sensitivity. For example, the $28^{\text {th }}$ order cladding mode of $83^{\circ}$-TFG exhibited an RI sensitivity of $156 \mathrm{~nm} / \mathrm{RIU}$, whereas the $43^{\text {rd }}$ mode of $72^{\circ}$-TFG displayed an RI sensitivity of $260 \mathrm{~nm} /$ RIU.

\section{2) RI sensitivity enhancement}

The theoretical analysis has verified that the RI sensitivity of cladding mode can be significantly enhanced with reduced cladding size. Thus, there are two ways to achieve high RI sensitivity for an Ex-TFG: a) directly inscribing grating in the thin cladding fiber [40]; b) reducing fiber cladding by using the hydrofluoric acid etching method [41]. In 2015, Yan et al. reported the RI sensitivity enhancement by inscribing the ExTFG in a thin cladding fiber with $40 \mu \mathrm{m}$ cladding radius, which has shown an RI sensitivity of $1180 \mathrm{~nm} / \mathrm{RIU}$ for the TM mode and $1150 \mathrm{~nm} / \mathrm{RIU}$ for the TE mode, respectively, compared to only $200 \mathrm{~nm} / \mathrm{RIU}$ and $170 \mathrm{~nm} / \mathrm{RIU}$ for the $62.5 \mu \mathrm{m}$ cladding 
radius fiber at the SRI of 1.345 (Fig.7a) [40]. In 2018, Luo et al. have reported a detailed investigation on increasing RI sensitivity of an Ex-TFG by using the etching method [41], achieving an average RI sensitivity, in the index region of 1.331.38, 6.3 times higher than that of the no-etched Ex-TFG, and the highest sensitivity has reached to $\sim 1600 \mathrm{~nm} / \mathrm{RIU}$ as the cladding diameter etched to $\sim 15 \mu \mathrm{m}$ (Fig. $7 \mathrm{~b}$ ).
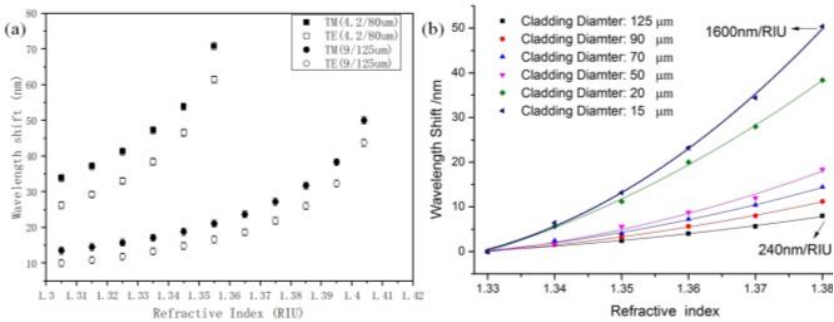

Fig. 7. (a) RI response of TM and TE peaks of $81^{\circ}-\mathrm{TFG}$ inscribed into SMF-28 fiber with $62.5 \mu \mathrm{m}$ cladding radius and SM1500 fiber with $40 \mu \mathrm{m}$ cladding radius [40]. (b) Resonance wavelength shifts for the TM mode peaks in etched fibers with different cladding radius sizes [41].

However, etching Ex-TFG will reduce the mechanical strength of the grating, thus difficult to keep it intact during the sensing process. In order to maintain the mechanical strength of grating, another method was proposed to enhance the RI sensitivity, i.e. coating the Ex-TFG with high refractive index material. In 2018, $\mathrm{Li}$ et al. have theoretically analyzed the RI sensitivity of $\mathrm{TiO}_{2}$ coated Ex-TFG, predicted such a coated grating may achieve an ultrahigh RI sensitivity of $32261.2 \mathrm{~nm} / \mathrm{RIU}$, which is 18.1 times higher than that of the uncoated structure [42].
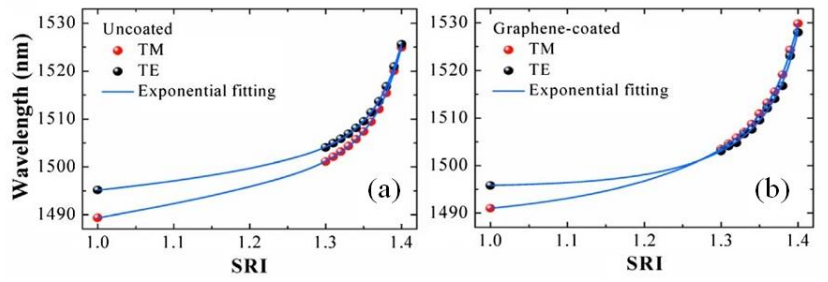

Fig. 8. RI sensing performance of Ex-TFG without (a) and with (b) graphene coating in SRI solutions [43].

Jiang et al. have experimentally demonstrated RI sensitivity enhancement by depositing high index nanomaterial graphene to the Ex-TFG and explored the spectrum and RI response [43]. Their results have shown the RI sensitivity of the graphenecoated Ex-TFG slightly increased in comparison with the bare Ex-TFG, seen in Fig. 8a and b. The effect in RI sensitivity enhancement using graphene coating is less due to the limited layers of graphene, whose RI decreases as the number of layers increases.

\section{3) Orientational RI response}

The RI sensing nature of a fiber grating is the interaction between surrounding medium and the grating evanescent field, which changes the effective mode index of resonance peak. The tilted structure of an Ex-TFG induces a non-circular symmetric field distribution of the $\mathrm{LP}_{1 \mathrm{~m}}$ cladding mode, which endows an orientation related RI response to the Ex-TFG. The resonant wavelength shift of a titled grating relies on the penetration depth of evanescent field of the cladding modes into the surrounding medium.

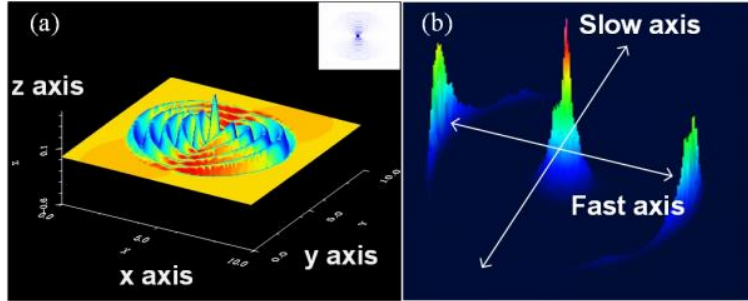

Fig. 9. Cladding mode distribution of Ex-TFG (a) Simulation results and (b) Captured near field results [29].

In 2020, Sun et al. have revealed the orientational RI responsivity of an Ex-TFG by analyzing the mode field distribution of cladding mode, which was verified in the experiment. This study pointed out that the Ex-TFG mainly excites the $\mathrm{LP}_{1 \mathrm{~m}}$ cladding mode, which has a non-circular symmetric evanescent field, thus resulting in orientational RI response [29]. Simulated results have shown that the $\mathrm{LP}_{1 \mathrm{~m}}$ cladding modes have orthogonal mode field distributions as depicted in Fig.9, and from the figure, we can see that there are two lobes along the fast axis of Ex-TFG, and the evanescent field power mainly concentrates in these two side lobes. This unique field distribution property promotes the Ex-TFG's orientational RI sensitivity.
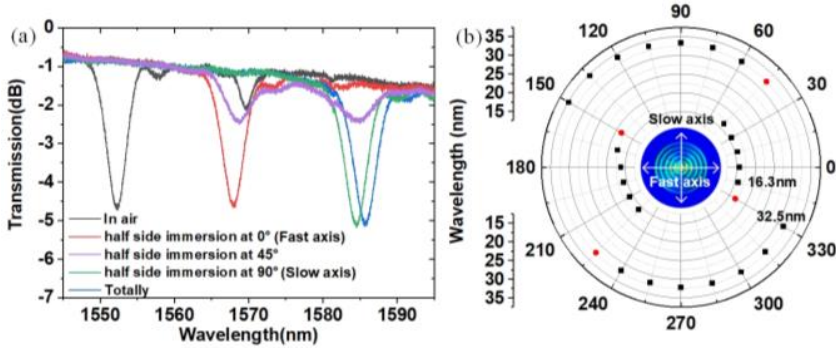

Fig. 10. Transmission spectra response of TM mode of Ex-TFG by sidely immersing $1.39 \mathrm{RI}$ oil (a) along $0^{\circ}, 45^{\circ}, 90^{\circ}$ and totally; (b) wavelength shift of TM cladding mode along different immersion angles with respect to the fast axis of grating from $0^{\circ}$ to $345^{\circ}[29]$.

To experimentally verify this orientational RI sensitivity, an Ex-TFG was immersed into an oil liquid in different directions. Figure 10a shows the spectra of the Ex-TFG when in the air or half side immersed in the oil (SRI $=1.39)$ at $0^{\circ}, 45^{\circ}$ and $90^{\circ}$. From the figure, we can see that the wavelength shift of the ExTFG immersing along the slow axis $\left(90^{\circ}\right)$ is almost twice than that along the fast axis $\left(0^{\circ}\right)$. Quantitatively, the resonance wavelength shift is about $16.3 \mathrm{~nm}$ around the fast axis and doubled to $32.5 \mathrm{~nm}$ around the slow axis in polar coordinates as shown in Fig. 10b. This unique orientational RI sensitivity reflects the birefringence and polarization function of the ExTFG, which can be utilized for vector sensing as discussed below.

\section{B. Temperature cross talk}

As reported in $[28,38]$, the Ex-TFGs have shown low thermal cross-talk, as the typical temperature sensitivities of the TM and TE modes are only about $6.8 \mathrm{pm} /{ }^{\circ} \mathrm{C}$ and $5.6 \mathrm{pm} /{ }^{\circ} \mathrm{C}$, respectively (Fig. 11 a). It was pointed out that the response of Ex-TFG to temperature depends on the cladding mode order [38]. The reported work in Ref. [38] shows the temperature sensitivity of the $40^{\text {th }}, 35^{\text {th }}, 31^{\text {st }}$ and $28^{\text {th }}$ cladding mode of four Ex-TFGs with different tilt angle $\left(75^{\circ}, 79^{\circ}, 81^{\circ}\right.$ and $\left.83^{\circ}\right)$ is measured as 
$4 \mathrm{pm} /{ }^{\circ} \mathrm{C}, 5.6 \mathrm{pm} /{ }^{\circ} \mathrm{C}, 6.8 \mathrm{pm} /{ }^{\circ} \mathrm{C}$ and $9 \mathrm{pm} /{ }^{\circ} \mathrm{C}$, respectively (Fig. 11b). These values are much lower than that of the typical LPGs (typically few hundred $\mathrm{pm} /{ }^{\circ} \mathrm{C}$ ) and also lower than standard FBGs $\left(\sim 10-12 \mathrm{pm} /{ }^{\circ} \mathrm{C}\right)$. The low temperature crosstalk property of Ex-TFGs is particularly desirable for high accuracy bio/chemical sensing applications.

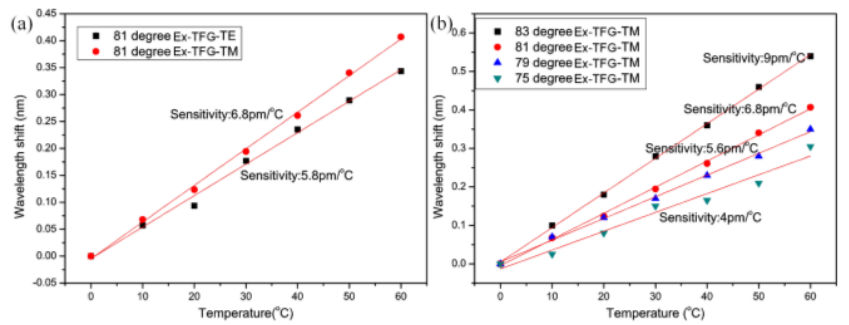

Fig. 11. The temperature response (a) for the TE and TM modes of an ExTFG with $81^{\circ}$ tilt angle and (b) for TM modes of Ex-TFGs of different titled angles at $75^{\circ}, 79^{\circ}, 81^{\circ}$ and $83^{\circ}[38]$.

\section{Sensing APPLiCATIONS OF EX-TFG}

Nowadays, due to the advantages of small size, high reliability and immune to electromagnetic interference, OFGs have been widely applied in structure monitoring, physical field measurement and bio/chemical detection. Owing to the high RI sensitivity, low thermal crosstalk and unique polarization property, Ex-TFGs have been particularly explored in the vector sensing and bio/chemical detection area.

\section{A. Vector sensors}

Most physical parameters have orientational information, usually, we need to employ multiple sensors in different directions to detect or monitor the changes along different directions, which increases the complexity and cost of the system. For an Ex-TFG, the intrinsic asymmetrical structure and uneven evanescent field distribution have made the ExTFG suit for vector sensing.

\section{1) Vector twist sensor}

The unique polarization dependent characteristics of Ex-TFG makes it possible to apply in twist sensing. In 2006, Chen et al. proposed a vector twist sensor based on an Ex-TFG with $81^{\circ}$ tilt angle [44], which can monitor both direction and amplitude of the torsion. The experimental results have shown that as the $81^{\circ}$-TFG is twisted in the clockwise direction from $0^{\circ}$ to $180^{\circ}$, the strength of the TM peaks decreases, and the TE increases, which indicates that the power is changing from one set of the polarization modes to the other set during the twist process, seen in Fig. 12a. By monitoring the intensity of resonance peaks, during twisting in clockwise and anti-clockwise, the TM and TE peaks show opposite trends, and the twist response is highly linear in the range of $-180^{\circ} \sim 180^{\circ}$ with $0^{\circ}$ as the turning point, which exhibits a twist sensitivity around $14.3 \mu \mathrm{W}(\mathrm{rad} / \mathrm{m})$. If the starting point of the twist sensor is set at either $\pm 90^{\circ}$, the direction and angle of the torsion can be inferred by observing the changes with resonance peaks of the two polarization states. This simple and low-cost twist-intensity demodulation technique makes Ex-TFG an excellent in-fiber vector twist sensor, which may be applied in the structure monitoring.
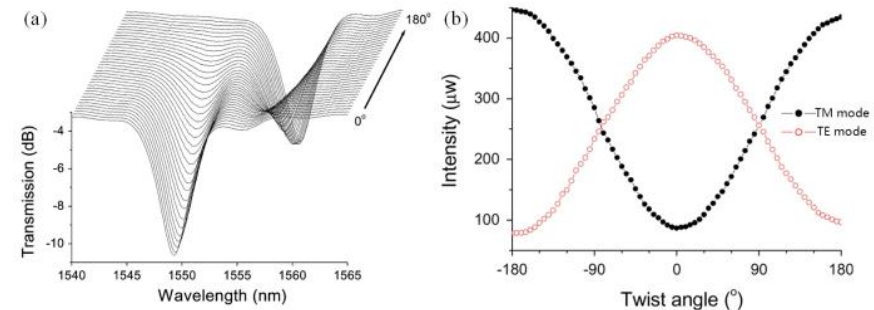

Fig. 12. The twist sensor based on an $81^{\circ}$-TFG: (a) the transmission spectra evolution from $0^{\circ}$ to $180^{\circ}$; (b) the intensity changes of the two polarization peaks under twist in clockwise and anti-clockwise directions [44].

\section{2) Vector loading sensor}

Loading sensors are demanded often in structural quality monitoring employed in measuring weight, direction and so on. Since the Ex-TFG structure is defined by the slow and fast axis, the mode effective index of cladding mode changes differently along the two axes when the Ex-TFG is subjected to loading. As loading applied along the slow axis of Ex-TFG, the birefringence induced by loading would coincide with that induced by the tilted grating structure, while the peaks of ExTFG would remain unchanged. Conversely, as loading applied along fast axis, the birefringent effect induced by loading and tilted grating structure would be cancelled each other out, and then the dual-peak turned into one peak. Such phenomenon offers an exceptional vector monitoring feature for the Ex-TFG. In 2009, Suo et al. reported a vector loading sensor by using an $81^{\circ}$-TFG [45]. In this experiment, transverse load is applied along the fast axis of grating, as seen in Fig. 13a. As increasing the loading, the intensity of the TM peak is decreasing and that of the TE peak is increasing (see Fig. 13b), and the intensity responses of TM and TE peaks have shown a good linear relationship with an approximately $2.04 \mu \mathrm{W} /\left(\mathrm{kg} \mathrm{m}^{-1}\right)$ sensitivity for the loading increasing from 3 to $31 \mathrm{~kg} / \mathrm{m}$ (seen in Fig. 13c). However, when the loading was applied to the grating slow axis $\left(\psi=90^{\circ}\right)$, the sensor almost did not respond to the perturbation (Fig. 13d). The transmitted light would be easily influenced when the load is applied to the fast axis and immuned as the loading applied along the slow axis. This distinguishing directional property makes an Ex-TFG desirable vector loading sensor to measure the amplitude and direction of an applied load at the same time. 

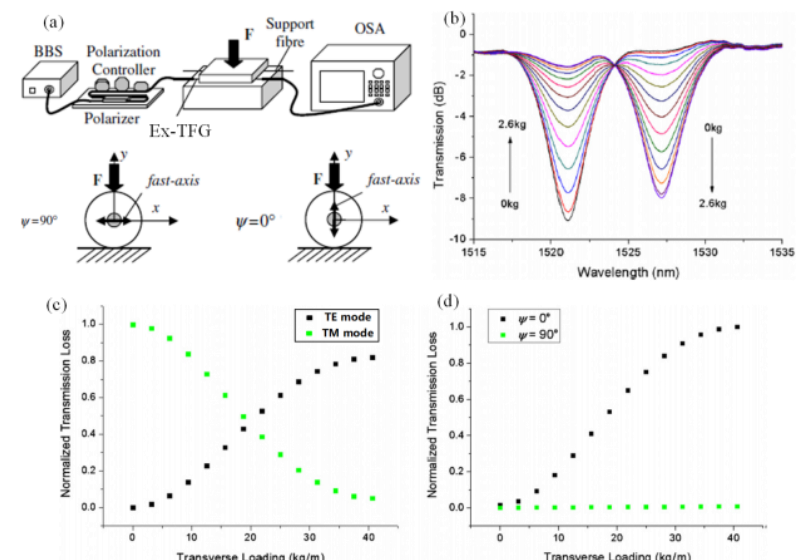

Fig. 13. The loading sensor based on an $81^{\circ}$-TFG: (a) experimental setup and schematic diagram of fast and slow axis; (b) The transmission spectra evolution from 0 to $2.6 \mathrm{~kg}$; (c) normalized transmission losses of the TM and TE modes plotted in linear scale with increasing load; (d) normalized transmission losses of the TM mode with the load applied to the fast axis $(\psi=0 \circ)$ and slow axis $\left(\psi=90^{\circ}\right)[45]$.

\section{3) Vector magnetometer}

Based on the direction-dependency RI response discussed previously, Lu et al. have proposed an Ex-TFG based vector magnetometer [46], for which the Ex-TFG is packaged within a magnetic fluid (MF) with highly dispersed ferromagnetic particle. Under the magnetic field, the random distribution of ferromagnetic particles becomes oriented along the magnetic field and forms magnetic chain clusters. By measuring the direction of magnetic chain clusters and the concentration of ferromagnetic particles, the strength and direction of the magnetic field could be obtained. The experimental results reported in Ref. [46] shows that the wavelength shift of the ExTFG has a polarized changing trend, indicated by a "figure-8shape", seen in Fig. 14a. The fast axis of the Ex-TFG shows the direction information of the magnetic field and the wavelength shift along the slow axis gives the strength information of the magnetic field. From Fig 14a and b, we can see the sensitivities to magnetic field orientation and intensity could reach as high as $0.41 \mathrm{~nm} / \mathrm{deg}$ and $2.45 \mathrm{~nm} / \mathrm{mT}$, respectively, and the detection limit of the magnetic field intensity is about $8.1 \mu \mathrm{T}$, which depends on the concentration of ferromagnetic particles in MF.

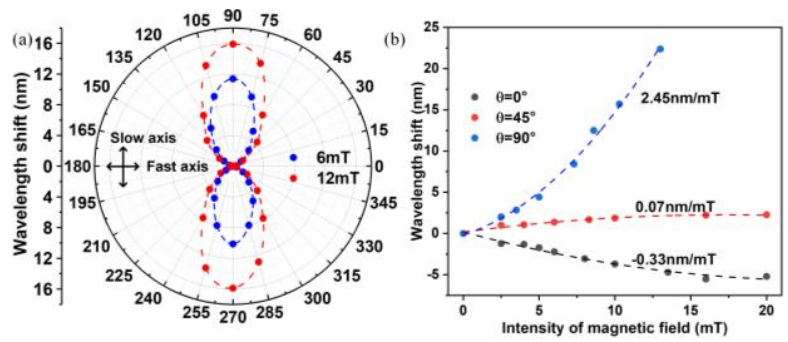

Fig. 14. (a) Wavelength shift as a function of magnetic field orientation in polar coordinate system under fixed magnetic field intensities as $6 \mathrm{mT}$ and $12 \mathrm{mT}$. (b)Wavelength shift versus magnetic field intensity with intersection angle $\theta$ of $0^{\circ}, 45^{\circ}$ and $90^{\circ}$ with respect to the fast axis of Ex-TFG [46].

\section{4) Vector accelerometer sensor}

In 2020 , Xie et al. have proposed an $81^{\circ}$-TFG based vector accelerometer sensor [47], in which the asymmetrical structure of the grating has shown a directionally dependent bend response. Based on its high axial strain sensitivities of -2.93 and $-2.31 \mathrm{pm} / \mu \varepsilon$ for the TE and TM cladding mode, and combined with the good orthotropic orientational bending sensitivities of the TE and TM modes, the vector accelerometer sensor exhibits strong orientation-dependent vibration properties, which also shows a "figure-8-shape" distribution for the vibration measurements at a certain frequency (Fig. 15d). The sensor has achieved a maximum acceleration sensitivity of $74.14 \mathrm{mV} / \mathrm{g}$ at $72 \mathrm{~Hz}$ and a maximum orientation sensitivity of $9.1 \mathrm{mV} / \mathrm{deg}$ for the TE cladding mode. The simple structure and stable performance of the Ex-TFG based vibration sensor could be suitable for the sensing and on-line monitoring of vibration measurement fields.

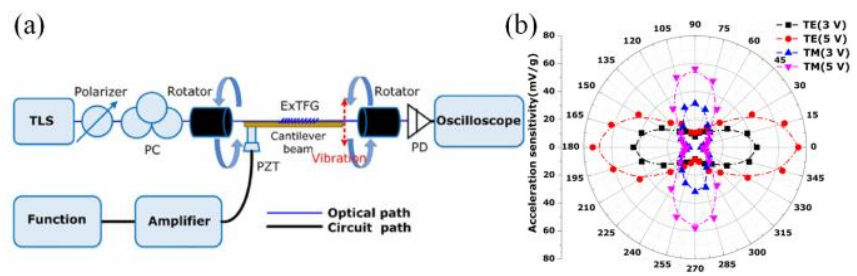

Fig. 15. (a) Wavelength shift as the variation of the axis strain of the Ex-TFG. (b) Acceleration sensitivity as a function of vibration orientation for the TE and TM modes at $72 \mathrm{~Hz}$ in a polar coordinate system under fixed driving voltages of 5 and $3 \mathrm{~V}$ [47].

\section{B. Ex-TFG based biochemical sensors}

With increasing demands of biomedical and biochemical detection technology, optical fiber sensing techniques have been attracting more and more attention in these fields due to their fast and simple test process. A vast number of researches about biochemical sensors in using LPGs and TFBGs have been reported [48-51, 15-18]. Comparing with LPGs, Ex-TFGs have high $\mathrm{Q}$ factor and lower thermal crosstalk, which are more suitable for biochemical sensing. Table I summarized the comparison of the different parameters of LPG, Ex-TFG and TFBG, in which the Ex-TFG showed a very high Figure of Merit (FoM) [15]. The higher FoM is the higher sensing precision. However, Ex-TFGs can only respond to the changing of SRI and cannot be used directly for bio/chemical sensing. To employ the Ex-TFGs in Bio/chemical sensing area, the outer surface of grating fiber needs to be bio-functionalized for biocompatibility and bio-selectivity [52-60].

TABLE I THE DIFFERENT PARAMETERS COMPARISON OF LPG, EX-TFG AND TFBG [15, 35, 37, 47, 48]

\begin{tabular}{ccccc}
\hline & Temperature & $\begin{array}{c}\text { Refractive } \\
\text { index }\end{array}$ & Bandwidth & $\begin{array}{c}\text { Figure of } \\
\text { Merit }\end{array}$ \\
\hline LPG & $70 \mathrm{pm} /{ }^{\circ} \mathrm{C}$ & $228 \mathrm{~nm} /$ RIU & $14.08 \mathrm{~nm}$ & 16.19 \\
Ex-TFG & $6 \mathrm{pm} /{ }^{\circ} \mathrm{C}$ & $821 \mathrm{~nm} / \mathrm{RIU}$ & $3.03 \mathrm{~nm}$ & 270.96 \\
TFBG & $0.35 \mathrm{pm} /{ }^{\circ} \mathrm{C}$ & $9.31 \mathrm{~nm} / \mathrm{RIU}$ & $0.5 \mathrm{~nm}$ & 18.62 \\
\hline \hline
\end{tabular}

\section{1) Humidity sensor}

Relative humidity $(\mathrm{RH})$ is a key parameter in environmental monitoring, weather forecasting and chemical applications. Jiang et al. have reported a graphene oxide (GO) deposited ExTFG for ultrafast humidity sensing and human breath monitoring [52]. Fig. 16a presents an RH sensor based on an Ex-TFG coated with GO that has a high surface-area-to-volume ratio and abundant functional groups to interact with water 
molecules. The experiment results have shown that both intensity and resonance wavelength of the RH sensor are linearly proportional to the change of $\mathrm{RH}$ in the range of $30 \%$ to $80 \%$, and exhibited the sensitivities of $0.027 \mathrm{~dB} / \% \mathrm{RH}$ for the former and $18.5 \mathrm{pm} / \% \mathrm{RH}$ for the latter, respectively. In addition, the Ex-TFG based RH sensor has demonstrated low hysteresis and stable RH response (shown in Fig. 16c). In order to evaluate the repeatability and response time, the RH sensor was employed to monitor a human breath with different breathing frequency. As a result of measurement and calculation, the RH sensor exhibited a response time of $42 \mathrm{~ms}$ and $115 \mathrm{~ms}$ recovery time (Fig. 16d), indicating good repeatability and reliability during the breathing cycles.
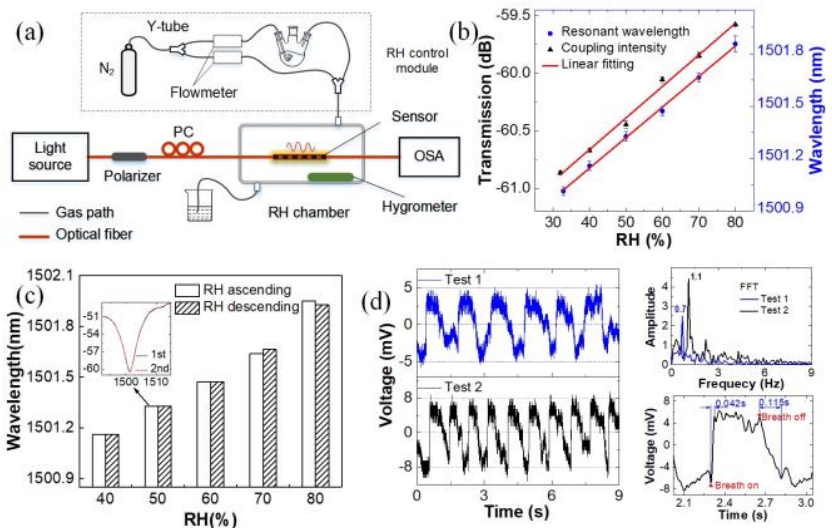

Fig. 16. (a) Experimental setup of GO-deposited Ex-TFG sensor for the RH response. (b) Resonant wavelength and intensity of Dip 1 versus the RH level. (c) Hysteresis test of the RH response, and the inset is the spectra of GO-deposited Ex-TFG sensor for repeated measurements at 50\%RH. (d) Responses of GO-deposited Ex-TFG sensor to human breathing [52].

\section{2) Heavy metal ion sensor}

Industrial wastewater usually contains a range of heavy metal ions such as $\mathrm{Pb}^{2+}, \mathrm{Cr}^{6+}, \mathrm{Hg}^{2+}$, etc., which would accumulate in the human body through the food chain and cause chronic poisoning. It is important to develop an effective technique to detect heavy metal ions.

In 2018, Liu et al. have reported a heavy metal ion sensor by employing an Ex-TFG assisted with black phosphorus (BP) film, to which the BP film was coated by layer-by-layer deposition technique on the outer surface of fiber (Fig. 17a) [53]. The key point is that BP film possesses superior molecular adsorption energy, which could adsorb heavy metal ions. The proposed BP functionalized sensor exhibited an ultrawide sensing range from $0.1 \mathrm{ppb}$ to $1.5 \times 10^{7} \mathrm{ppb}$ and a non-linear sensitivity up to $0.5 \times 10^{-3} \mathrm{~dB} / \mathrm{ppb} @ 1.5 \times 10^{7} \mathrm{ppb}$ (see in Fig. 17b). Another potential advantage of the reported heavy metal biosensor is that the limit of detection is as low as $0.25 \mathrm{ppb}$ for $\mathrm{Pb}^{2+}$ ions, which is only one-fortieth of WHO's permissible limit of $10 \mathrm{ppb}$ for lead in drinking water. This study provides an optical platform for high sensitivity detection of heavy metal with ultralow limits.

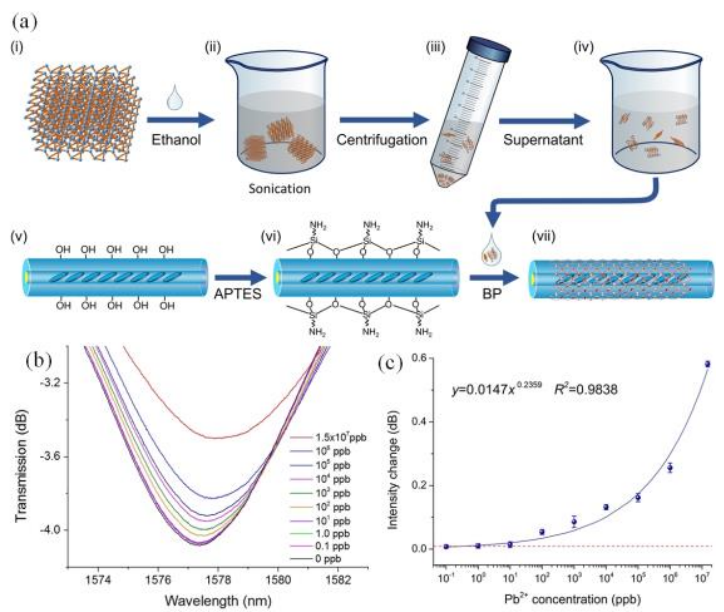

Fig. 17 (a) Schematic representation of synthesis of BP nanosheets (i-iv) and deposition process of BP nanosheets on fiber device surface (v-vii); (b) Resonant intensity change against $\mathrm{Pb} 2+$ concentrations;(c) Intensity change versus the concentration of $\mathrm{Pb}^{2+}[53]$.

\section{3) Glucose sensor}

Glucose is the energy source and metabolism intermediate product of living cells, which is the main energy supply material of living beings. Too much glucose would lead to obesity and diabetes, and too little would cause hypoglycemia. So, it is important to detect the glucose concentration. In 2014, Luo et al. [54] proposed a novel selective glucose sensor based on an Ex-TFG modified by glucose oxidase (GOD), in which GOD is a specific catalyze enzyme that could promote glucose converting to the glucose acid and lead to the changing of RI, and the Ex-TFG could detect very weak various of RI. Before sensing, the GOD was immobilized to the outer surface of fiber cladding by using the aminopropyl triethoxysilane (APTES). As shown in Fig.18a, the response of the sensor to the glucose concentration showed a linear relationship with $0.298 \mathrm{~nm} /(\mathrm{mg} / \mathrm{mL})$ sensitivity in the range of $0 \sim 0.3 \mathrm{mg} / \mathrm{mL}$. Furthermore, in 2016, they have achieved the sensitivity of $1.514 \mathrm{~nm} /(\mathrm{mg} / \mathrm{mL})$ for the glucose concentration from 0.1 to
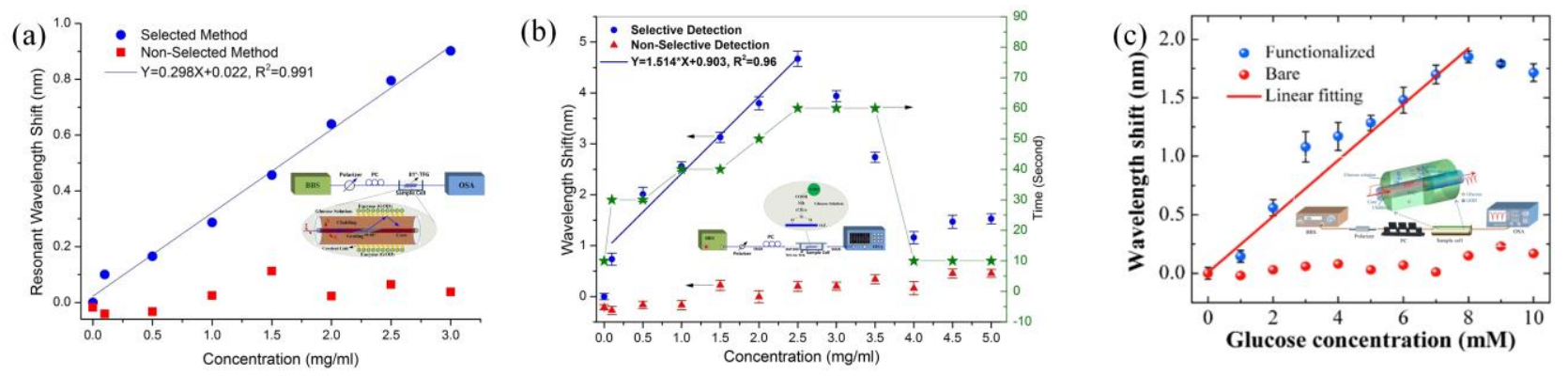

Fig. 18 (a) The schematic of GOD-immobilized Ex-TFG and sensing performance of D-glucose concentration [54]. (b) Experimental setup for the detection of the glucose concentration using thin cladding Ex-TFG and the sensing performance of glucose concentration from 
TABLE II LABEL-FREE IMMUNOSENSOR

\begin{tabular}{|c|c|c|c|c|c|}
\hline Analyte & Sensing materials & Sensitivity & Detection range & LOD & Ref \\
\hline $\mathrm{PCV} 2$ & anti-PCV2 MAbs & & $0-2.08 \times 10^{3} \mathrm{ng} / \mathrm{mL}$ & $4.06 \mathrm{ng} / \mathrm{mL}$ & {$[57]$} \\
\hline NT-proBNP & anti-NT-proBNP MAbs & $45.967 \mathrm{pm} /(\mathrm{ng} / \mathrm{mL})$. & $0-1.0 \mathrm{ng} / \mathrm{mL}$ & $0.5 \mathrm{ng} / \mathrm{mL}$ & {$[58]$} \\
\hline NDV & anti-NDV MAbs & $1.627 \mathrm{pm} /(\mathrm{pg} / \mathrm{mL})$ & $0-200 \mathrm{pg} / \mathrm{mL}$ & $25 \mathrm{pg} / \mathrm{mL}$ & {$[59]$} \\
\hline sPD-L1 & anti-sPD-L1 MAbs & & $0.0-1000 \mathrm{ng} / \mathrm{mL}$ & $5 \mathrm{ng} / \mathrm{mL}$ & [60] \\
\hline
\end{tabular}

$2.5 \mathrm{mg} / \mathrm{mL}$ by using Ex-TFG fabricated in a thin cladding fiber (Fig. 18b) [55]. In 2018, Jiang et al. reported GO coated ExTFG based glucose sensor, in which the APTES is replaced by GO coating [56]. The GO coating could not only absorb the GOD, but also enhance the sensitivity of Ex-TFG. The experiment results show that the GO coated Ex-TFG sensor could directly sense the glucose concentration in DI water without the need for a special buffered solution, which has shown a sensitivity of $0.25 \mathrm{~nm} / \mathrm{mM}$ with a $0-8 \mathrm{mM}$ detection range (Fig. 18c).

\section{4) Label-free immunosensor}

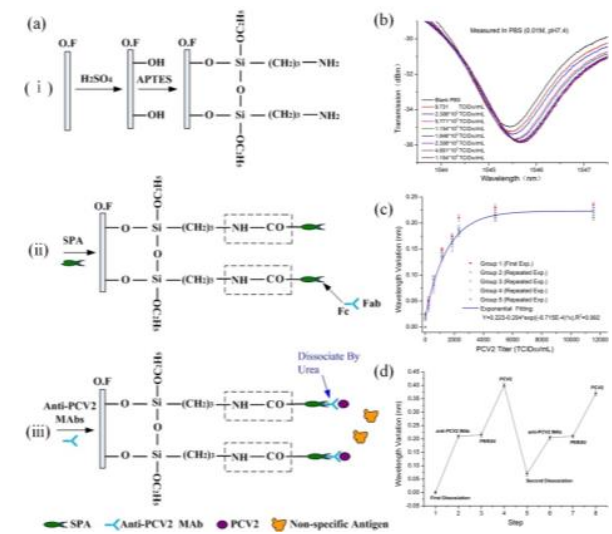

Fig. 19 (a) Mechanism of chemical linkage of APTES, SPA, and antiPCV2 Mab on the fiber surface. (b) Spectrum evolution for the first group of PCV2 detection. (c) Resonance wavelength variation of the first group (Group1) and repeated groups of PCV2 detection (Groups2-5), and the fitting curve (blueline) of the relation between the wavelength variation and PCV2 titer. Relative standard deviation for 5 different measurements: $0.038<\operatorname{RSD}<0.15$. (d) Variations of resonance wavelength for the first and second specificity experiments of the sensor [57].

Recently, label-free immunosensors for biomedical sensing applications are extensively studied, which is based on specific interactions between antibodies and antigens. In 2016, Luo et al. conducted a research [57] on specific detection of porcine circovirus type 2 (PCV2), which is a single stranded DNA virus of mammals and the primary etiological agent of many diseases. In the experiment, the Ex-TFG was immobilized with antiPCV2 monoclonal antibodies (MAb) through staphylococcal protein A (SPA) that acts as the probe to detect the PCV2, and the detailed detection process can be seen in Fig. 19a. Once the PCV2 combined with SPA immobilized Ex-TFG, the resonance wavelength of Ex-TFG will redshift. The experiment results show that the immunosensor exhibited an obvious response from 0 to $1.154 \times 10^{4} \mathrm{TCID}_{50} / \mathrm{mL}$ and the limit of detection (LOD) is $9.371 \mathrm{TCID}_{50} / \mathrm{mL}$ (Fig. 19b, c). Meanwhile, the immunosensor also performed an excellent specificity for the PCV2 detection, as shown in Fig. 19d. By further exploring the Ex-TFG sensing capability, Luo' s group has presented another highly specific and fast detection of human N-terminal pro-Btype natriuretic peptide (NT-proBNP) biosensor with a detection LOD of $0.5 \mathrm{ng} / \mathrm{mL}$, which could be applied in early heart failure diagnosis [58]. Furthermore, Luo et al. have improved the detection limit and sensitivity of the Ex-TFG based immunosensor by employing localized surface plasmon resonance effect. In the experiment, the gold nanospheres and gold nanoshells were coated to the fiber surface [59]. Based on this, they have achieved Newcastle disease virus (NDV) and soluble programmed death ligand-1 detection, which would be potentially applied in the clinic for the fast and early diagnosis of NDV epidemic disease of poultry and human tumor, respectively [60]. The sensing performance of Ex-TFG based label-free immunosensors developed by Luo et al. is summarized in Table II.

\section{CONCLUSION AND OUTLOOK}

In conclusion, Ex-TFGs have been developed rapidly in recent years, especially in various sensing fields. In this paper, we have provided a detailed review on the recent development of Ex-TFGs, including the theoretical and experimental studies, and technical developments and applications. Ex-TFGs have the same mode coupling mechanism as LPGs, in which the core mode is coupled into forward propagating cladding modes, but the asymmetric structure of the Ex-TFG makes the core-tocladding coupling into $\mathrm{LP}_{1 \mathrm{~m}}$ modes with two orthogonal polarization state (TE and TM polarization state), respectively. This exceptional polarization property has donated the unique vector sensing capability to the Ex-TFGs, enabling them to be developed as bending, loading, twist and magnetic sensors, capable of measuring not just amplitude but also the direction recognition of the physical parameters. Furthermore, Ex-TFGs have exhibited significantly high RI sensitivity and low thermal crosstalk, which provides an optical fiber probe platform for a wide range of environmental monitoring and biomedical detection applications. Taking the advantages of high RI responsivity, unique vector sensing capability, simple fiber probe structure and good reproducibility, we could foresee ExTFGs become a mainstream of optical fiber devices to be developed and utilized for applications in vector and biochemical sensing. Furthermore, the demonstrated success of integration with the nano and bio-functional materials offers a great potential to explore Ex-TFGs as ultrasensitive and high selectivity biosensors for environment monitoring, new drug development, medical diagnosis and healthcare.

\section{REFERENCES}

[1]. G. Meltz, W. Morey, W.H. Glenn, "Formation of Bragg 
gratings in optical fibers by a transverse holographic method," Opt. Lett. vol., 14, pp. 823-825, 1989.

[2]. T. Erdogan, "Fiber Grating Spectra," J. Lightwave Technol., vol. 15, no. 8, pp. 1277-1294, 1997.

[3]. T. Erdogan, "Cladding-mode resonances in short- and long-period fiber grating filters," J. Opt. Soc. Am. A., vol. 14, no. 8, pp. 1760-1773, 1997.

[4]. KS. Lee, T. Erdogan, "Fiber mode coupling in transmissive and reflective tilted fiber gratings," Appl. Opt., vol. 39, no. 9, pp. 1394-1404, 2000.

[5]. R. Essiambre and R. W. Tkach, "Capacity Trends and Limits of Optical Communication Networks," in Proceedings of the IEEE, vol. 100, no. 5, pp. 1035-1055, 2012,

[6]. X. Feng, Y. Liu, S. Fu and X. Dong, "Switchable dualwavelength ytterbium-doped fiber laser based on a fewmode fiber grating," IEEE Photonics Technol. Lett., vol. 16, no. 3, pp. 762-764, 2004.

[7]. M. Yin, B. Gu, Q. An, et al. "Recent development of fiberoptic chemical sensors and biosensors: Mechanisms, materials, micro/nano-fabrications and applications," Coord. Chem. Rev., vol. 376, no. 1, pp. 348-392, 2018.

[8]. K.O. Hill, et al., "Photosensitivity in optical fiber waveguides: Application to reflection filter fabrication," Appl. Phys. Lett., vol. 32, no. 10, pp. 647-649, 1978.

[9]. I.M. Littler, M. Rochette, and B. Eggleton, "Adjustable bandwidth disperionless bandpass FBG optical filter," Opt. Exp., vol. 13, pp. 3397-3407, 2005.

[10]. T.A. Berkoff and A.D. Kersey, "Fiber Bragg grating array sensor system using a bandpass wavelength division multiplexer and interferometric detection," IEEE Photonics Technol. Lett., vol. 8, no. 11, pp. 1522-1524, 1996.

[11]. A.M. Vengsarkar, V. Bhatia, T. Erdogan, et al., "Longperiod fiber gratings as band-rejection filters," $J$. Lightwave Technol., vol. 14, pp. 58-65, 1996.

[12]. F. Chiavaioli, F. Baldini, C. Trono, S. Jana, S. Tombelli, et al., "Sol-Gel-Based Titania-Silica Thin Film Overlay for Long Period Fiber Grating-Based Biosensors." Anal. Chem., vol. 87, no. 24, pp. 12024-12031,2015.

[13]. T. Guo, H. Tam, P. Krug, and J. Albert, "Reflective tilted fiber Bragg grating refractometer based on strong cladding to core recoupling," Opt. Exp., vol. 17, pp. 57365742, 2009

[14]. Z. Yan, A. Adedotun, K. Zhou, L. Zhang, "Highly sensitive temperature and strain sensors based on all-fiber 45-TFG Lyot filter," Proc. SPIE 8439, Optical Sensing and Detection II, 843905, 2012.

[15]. J. Albert, L. Shao, and C. Caucheteur, "Tilted fiber Bragg gratings sensors," Laser Photon. Rev., vol. 7, no. 1, pp. 83-108, 2013

[16]. T. Guo, F. Liu, B. Guan, and J. Albert, "Tilted fiber grating mechanical and biochemical sensors," Opt. Laser Technol., vol. 78, pp. 19-33, 2016.

[17]. M. Loyez, J Albert, C. Caucheteur and R. Wattiez, "Cytokeratins Biosensing Using Tilted Fiber Gratings," Biosensors., vol. 8, no.3, pp. 74-81, 2018.
[18]. T. Guo, F. Liu, B. Guan, and J. Albert, "Polarimetric multi-mode tilted fiber grating sensors," Opt. Exp., vol. 22, pp. 7330-7336, 2014.

[19]. P.S. Westbrook, T.A. Strasser, "In-line polarimeter using blazed fibre gratings," IEEE Photonics Technol. Lett., vol. 12, pp. 1352-1354, 2000.

[20]. K. Zhou, G. Simpson, X. Chen, L. Zhang, and I. Bennion, "High extinction ratio in-fiber polarizers based on $45^{\circ}$ tilted fiber Bragg gratings," Opt. Lett., vol. 30, pp. 12851287, 2005.

[21]. Z. Yan, K. Zhou, L. Zhang, "In-fiber linear polarizer based on UV-inscribed $45^{\circ}$ tilted grating in polarization maintaining fiber," Opt. Lett., vol. 37, no. 18, pp. 3819$3821,2012$.

[22]. H. Qin, Z. Yan, L. Zhang, et al., "In-fiber singlepolarization diffraction grating based on radiant tilted fiber grating," Opt. Lett., vol. 44, pp. 4407-4410, 2019.

[23]. T. Wang, Z. Yan, C. Mou, Z. Liu, Y. Liu, K. Zhou, et al., "Narrow bandwidth passively mode locked picosecond Erbium doped fiber laser using a $45^{\circ}$ tilted fiber grating device," Opt. Exp., vol. 25, no. 14, pp. 16708-16714, 2017.

[24]. G. Wang, L. Zhang, et al., "Highly Efficient Optical Beam Steering Using an In-Fiber Diffraction Grating for Full Duplex Indoor Optical Wireless Communication," $J$. Lightwave Technol., vol. 36, no. 19, pp. 4618-4625, 2018.

[25]. G. Wang, C. Wang, Z. Yan, and L. Zhang, "Highly efficient spectrally encoded imaging using a $45^{\circ}$ tilted fiber grating," Opt. Lett., vol. 41, no.11, pp. 2398-2401, 2016.

[26]. K. Zhou, L. Zhang, X. Chen, and I. Bennion, "Optic sensors of high refractive-index responsivity and low thermal cross sensitivity that use fiber Bragg gratings of $>80^{\circ}$ tilted structures," Opt. Lett., vol. 31, no.9, pp. 1193-1195, 2006.

[27]. C. Mou, Z. Yan, K. Zhou, and L. Zhang, "Optical fibre sensors based on UV inscribed excessively tilted fibre grating," in Optical sensors: new developments and practical applications., Mohamad Yasin, Sulaiman Wadi Harun and Hamzah Arof, IntechOpen, 2014, pp.23-46. [Online]Available: https://www.intechopen.com.

[28]. K. Zhou, L. Zhang, X. Chen, and I. Bennion, "Low Temperature Sensitivity Grating Devices Based on Ex-45 Tilting Structure Capable of Forward-Propagating Cladding Modes Coupling," J. Lightwave Technol., 24, 5087-5094 (2006).

[29]. Y. Sun, T. Lu, H. Wang, Q. Sun, Z. Yan, L. Zhang, et al., "Theoretical and Experimental Analysis of the Directional RI Sensing Property of Tilted Fiber Grating," J. Lightwave Technol., doi: 10.1109/JLT.2020.3027947.

[30]. KS. Lee, T. Erdogan, "Fiber mode conversion with tilted gratings in an optical fiber," J. Opt. Soc. Am. A., vol. 18, no. 5, pp. 1176-1185, 2001.

[31]. G. Yin, S. Lou, Q. Li, and H. Zou, "Theory analysis of mode coupling in tilted long period fiber grating based on the full vector complex coupled mode theory," Opt. Laser Technol., vol. 48, no. 6, pp. 60-66, 2013.

[32]. L. Dong, B. Ortega, and L. Reekie, "Coupling 
characteristics of cladding modes in tilted optical fiber Bragg gratings," Appl. Opt., vol. 37, no. 22, pp. 5099-5105, 1998.

[33]. Z. Li, J. Shen, Q. Ji, X. Ruan, Y. Zhang, Y. Dai, and Z. Cai, "Tuning the resonance of polarization-degenerate LP1, 1 cladding mode in excessively tilted long period fiber grating for highly sensitive refractive index sensing," J. Opt. Soc. Am. A., vol. 35, pp. 397-405, 2018.

[34]. M.Z. Alam and J. Albert, "Selective Excitation of Radially and Azimuthally Polarized Optical Fiber Cladding Modes," J. Lightwave Technol., vol. 31, pp. 3167-3175, 2013.

[35].Z. Yan, H. Wang, L. Zhang, et al., "Theoretical and experimental analysis of excessively tilted fiber gratings," Opt. Exp. vol. 24, pp. 12107-12115, 2016.

[36]. K.O. Hill, B. Malo, J. Albert, "Bragg gratings fabricated in monomode photosensitive optical fibre by UV exposure through a phase mask," Appl. Phys. Lett., vol. 62, pp. 1035-1037, 1993.

[37]. D.Z. Anderson, V. Mizrahi, "Production of in-fibre gratings using a diffractive optical element," Electron. Lett., vol. 29, pp. 566-567, 1993.

[38]. Z. Yan, Q. Sun, C. Wang, Z. Sun, C. Mou, K. Zhou, D. Liu, L. Zhang, "Refractive index and temperature sensitivity characterization of excessively tilted fiber grating," Opt. Exp., vol. 25, pp. 3336-3346, 2017.

[39]. C. Mou, K. Zhou, Z. Yan, H. Fu, L. Zhang, "Liquid level sensor based on an excessively tilted fibre grating." Opt. Commun., vol. 305, pp. 271-275, 2013.

[40]. Z. Yan, Z. Sun, K. Zhou, L. Zhang, et al., "Numerical and Experimental Analysis of Sensitivity-Enhanced RI Sensor Based on Ex-TFG in Thin Cladding Fiber,' J. Lightwave Technol., vol. 33, no. 14, pp. 3023-3027, 2015.

[41]. H. Lu, B. Luo, et al., "Study on spectral and refractive index sensing characteristics of etched excessively tilted fiber gratings," Appl. Opt., vol. 57, no.10, pp. 2590-2596, 2018.

[42]. Z. Li, Z. Yu, B. Yan, Y. Dai, et al., "Theoretical analysis of tuning property of the graphene integrated excessively tilted fiber grating for sensitivity enhancement," J. Opt. Soc. Am. B., vol. 36, pp. 108-118, 2019.

[43]. B. Jiang, G. Yin, K. Zhou, C. Wang, X. Gan, J. Zhao, L. Zhang, "Graphene-induced unique polarization tuning properties of excessively tilted fiber grating," Opt. Lett., vol. 41, no. 23, pp. 5450-5453, 2016.

[44]. X. Chen, K. Zhou, L. Zhang and I. Bennion, "In-Fiber Twist Sensor Based on a Fiber Bragg Grating With $81^{\circ}$ Tilted Structure," IEEE Photonics Technol. Lett., vol. 18, no. 24, pp. 2596-2598, 2006.

[45]. R. Suo, X. Chen, K. Zhou, L. Zhang and I. Bennion, "Infibre directional transverse loading sensor based on excessively tilted fibre Bragg gratings," Meas. Sci. Technol., vol. 20, no. 3, pp. 034015-034020, 2009.

[46]. T. Lu, Y. Sun, Y. Moreno, Z. Yan, L. Zhang, et al., "Excessively tilted fiber grating-based vector magnetometer," Opt. Lett., vol. 44, no.10, pp. 2494-2497, 2019.
[47]. L. Xie, B. Luo, L. Zhang, et al., "Orientation-dependent optic-fiber accelerometer based on excessively tilted fiber grating," Opt. Lett., vol. 45, pp. 125-128, 2020.

[48]. S.W. James, R.P. Tatam, "Optical fibre long-period grating sensors: characteristics and application," Meas. Sci. Technol., vol.14, no.5, 2003.

[49]. C. Francesco, G. Carlos, J. Pedro, et al., "Towards a Uniform Metrological Assessment of Grating-Based Optical Fiber Sensors: From Refractometers to Biosensors," Biosensors., vol.7, no. 4, pp.23-51, 2017.

[50]. F. Chiavaioli, F. Baldini, S. Tombelli, C. Trono, A. Giannetti, "Biosensing with optical fiber gratings," Nanophotonics., vol. 6, no. 4, pp.663-679, 2017.

[51]. S. Cai, F. Liu, R. Wang, Y. Xiao, K. Li, C. Caucheteur and T. Guo, "Narrow bandwidth fiber-optic spectral combs for renewable hydrogen detection," Sci. China-Inf. Sci., doi: 10.1007/s11432-020-3058-2.

[52]. B. Jiang, Z. Bi, Z. Hao, Q. Yuan, D. Feng, K. Zhou, et al., "Graphene oxide-deposited tilted fiber grating for ultrafast humidity sensing and human breath monitoring," Sens. Actuator B-Chem., vol. 293, pp. 336-341, 2019.

[53]. C. Liu, Z. Sun, L. Zhang, J. Lv, X. Yu, L. Zhang, and X. Chen, "Black phosphorus integrated tilted fiber grating for ultrasensitive heavy metal sensing," Sens. Actuator BChem., vol.257, pp. 1093-1098, 2018.

[54]. B. Luo, Z. Yan, Z. Sun, J. Li, et al., "Novel glucose sensor based on enzyme-immobilized $81^{\circ}$ tilted fiber grating," Opt. Exp., vol.22, no. 25, pp. 30571-8, 2014.

[55]. B. Luo, Z. Yan, Z. Sun, Y. Liu, M. Zhao, and L. Zhang, "Biosensor based on excessively tilted fiber grating in thin-cladding optical fiber for sensitive and selective detection of low glucose concentration." Opt. Exp., vol. 23, no. 25, pp. 32429-32440, 2015.

[56]. B. Jiang, K. Zhou, C. Wang, Q. Sun, Y. Guo, Z. Tai, et al., "Label-free glucose biosensor based on enzymatic graphene oxide-functionalized tilted fiber grating," Sens. Actuator B-Chem., vol. 254, pp. 1033-1039, 2018.

[57]. B. Luo, S. Wu, W. Zou, et al., "Label-free immunoassay for porcine circovirus type 2 based on excessively tilted fiber grating modified with staphylococcal protein A," Biosens. Bioelectron., vol. 86, pp. 1054-1060, 2016.

[58]. B. Luo, S. Wu, L. Zhang, et al., "Human heart failure biomarker immunosensor based on excessively tilted fiber gratings," Biomed. Opt. Express., vol. 8, no.1, pp. 57-67, 2017.

[59]. B. Luo, Y. Xu, S. Wu, et al., "A novel immunosensor based on excessively tilted fiber grating coated with gold nanospheres improves the detection limit of Newcastle disease virus," Biosens. Bioelectron., vol.100, pp. 169$175,2017$.

[60]. B. Luo, Y. Wang, H. Lu, S. Wu, Y. Lu, et al., "Label-free and specific detection of soluble programmed death ligand-1 using a localized surface plasmon resonance biosensor based on excessively tilted fiber gratings," Biomed. Opt. Express., vol. 10, no.10, pp. 5136-5148, 2019. 